\title{
NEW MODELS FOR THE SPECTRAL EVOLUTION OF GALAXIES
}

\author{
GUSTAVO BRUZUAL A. \\ Centro de Investigaciones de Astronomla, CIDA \\ Apartado Postal 264, Mérida 5101-A, Venezuela
}

Bruzual \& Charlot (1991, BC91 hereafter) have supplemented the purely photometric isochrone synthesis models of Charlot \& Bruzual (1991) with an updated library of stellar spectra and evolutionary tracks in order to compute the spectral evolution of stellar populations. BC91 used the evolutionary tracks of Maeder \& Meynet (1989), with the main-sequence lifetimes for stars in the mass range $1.3 M_{\odot} \leq m \leq 2.5 M_{\odot}$, revised as prescribed by Maeder \& Meynet (1991). BC91 included an accurate description of the late evolution of low and intermediatemass stars as hot nuclei of planetary nebulae and through the white dwarf cooling sequence. While stars in these stages contribute negligibly to the optical/near-IR light, the self-consistent model of BC91 for an initial burst of star formation can account for the UV excess observed in quiescent early-type galaxies. Models that do not include post-AGB stars require additional star formation to account for this UV excess.

The library of stellar spectra assembled by BC91 includes observed near-IR spectra from $1.22 \mu \mathrm{m}$ to $2.56 \mu \mathrm{m}$ of stars in a wide range of spectral types and luminosity classes. The assignment of observed spectra to stars at different positions along the evolutionary tracks in the HR diagram is done by $\mathrm{BC} 91$ taking into account the full range of optical/near-IR standard star calibrations. This insures a better representation of the light at all wavelengths than in models in which the spectra are selected from the $B-V$ color or the effective temperature of the stars alone. BC91 supplemented their spectral library with model atmospheres of very hot and very cool stars for which observed spectra were not available, and used appropriate color calibrations for stars with circumstellar shells and dusty envelopes.

The isochrone synthesis spectral evolution models of BC91 reproduce the observed spectra of nearby galaxies with an accuracy that was previously achieved only by spectral synthesis techniques and introduce a number of significant improvements over earlier work. The models based on this revised set of tracks and stellar spectra confirm and even improve the successful comparison with observations presented by Charlot \& Bruzual (1991).

\section{References}

Bruzual A., G., \& Charlot, S. 1991, ApJ, submitted

Charlot, S., \& Bruzual A., G. 1991, ApJ, 367, 126

Maeder, A., \& Meynet, G. 1989, A\&A, 210, 155

Maeder, A., \& Meynet, G. 1991, private communication 